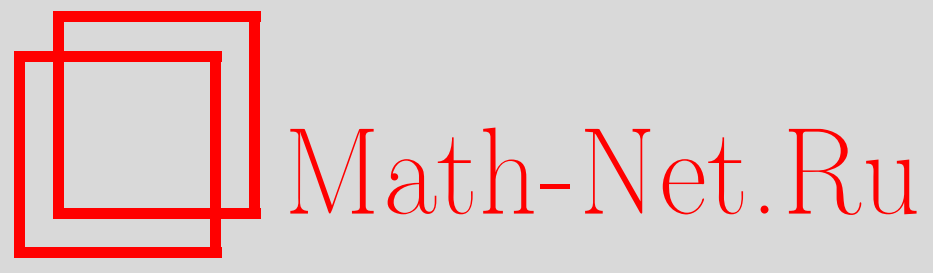

Л. К. Ширяева, Е. Г. Репина, О некоторых свойствах симметричной копулы Граббса, Вестн. Сам. гос. техн. ун-та. Сер. Физ.-мат. науки, 2018, номер 4, 714-734

DOI: https://doi.org/10.14498/vsgtu1640

Использование Общероссийского математического портала MathNet.Ru подразумевает, что вы прочитали и согласны с пользовательским соглашением

http://www . mathnet.ru/rus/agreement

Параметры загрузки:

IP: 54.84 .234 .179

26 апреля 2023 г., 05:50:51

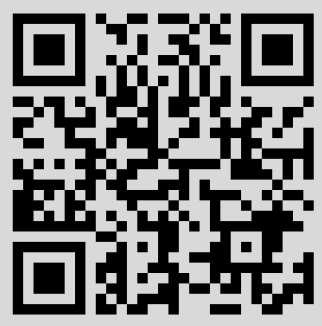


Вестн. Сам. гос. техн. ун-та. Сер. Физ.-мат. науки. 2018. Т. 22, № 4 . С. 714-734 ISSN: 2310-7081 (online), 1991-8615 (print)

УДК 519.24

\title{
О некоторых свойствах симметричной копулы Граббса
}

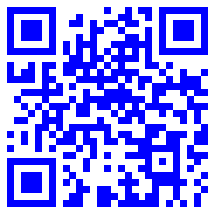

\section{Л. К. Ширяева, Е. Г. Репина}

Самарский государственный экономический университет, Россия, 443090, Самара, ул. Советской Армии, 141.

\begin{abstract}
Аннотация
Изучаются односторонние статистики Граббса, т. е. экстремальные стьюдентизированные отклонения наблюдений от выборочного среднего, найденные по нормально распределенной выборке. Исследуется двупараметрическое совместное распределение этих статистик, возникающее в случае, когда присутствующее в выборке аномальное наблюдение (выброс) отличается от остальных наблюдений величиной дисперсии. Выводится формула для вычисления плотности распределения вероятностей стьюдентизированного отклонения выброса от среднего. Из совместного распределения статистик Граббса извлекается двупараметрическая копула Граббса. Доказывается, что эта копула является симметричной. Как следствие, односторонние статистики Граббса обладают свойством обмениваемости. Выполняется компьютерное моделирование скаттерплотов из копулы Граббса. Анализ скаттерплотов показывает, что статистическая зависимость, описываемая копулой Граббса, является отрицательной. Для исследования влияния параметров копулы на силу этой зависимости выполняется оценивание коэффициента ранговой корреляции $\tau$-Кендалла копулы. Алгоритм оценивания использует компьютерное моделирование и реализован в пакете R. Найдено, что параметры копулы $n$ и $\nu>0$ оказывают разнонаправленное влияние на величину коэффициента $\tau$-Кендалла. Так, рост параметра $n$ при неизменной величине параметра $\nu$ приводит к уменьшению (по абсолютной величине) коэффициента $\tau$-Кендалла, что отражает уменьшение силы взаимосвязи между маргиналами, заключенными в копулу Граббса. Если не изменять параметр $n$, то с увеличением параметра $\nu$ до значений, близких к 1, коэффициент $\tau$-Кендалла уменьшается (по абсолютной величине), что отражает уменьшение силы взаимосвязи. Дальнейший рост параметра $\nu$ приводит к росту коэффициента $\tau$-Кендалла (по абсолютной величине), что отражает усиление отрицательной взаимозависимости между маргиналами.
\end{abstract}

\section{Научная статья}

ə () (7) Контент публикуется на условиях лицензии Creative Commons Attribution 4.0 International (https://creativecommons.org/licenses/by/4.0/deed.ru)

\section{Образец для цитирования}

Ши ряева Л. К., Репин а Е. Г. О некоторых свойствах симметричной копулы Граббса // Вестн. Сам. гос. техн. ун-та. Сер. Физ.-мат. науки, 2018. Т. 22, № 4. С. 714-734. doi: $10.14498 /$ vsgtu1640.

\section{Сведения об авторах}

Людмила Константиновна Ширяева (10) http://orcid.org/0000-0003-2890-1786 кандидат физико-математических наук, доцент; доцент каф. статистики и эконометрики; e-mail: Shiryeva_LK@mail.ru

Евгения Геннадъевна Репина (1) http://orcid.org/0000-0001-7151-3266 кандидат экономических наук, доцент; доцент каф. статистики и эконометрики; e-mail: violet261181@mail.ru 
Ключевые слова: совместная функция распределения стандартизированных минимума и максимума, выброс, нормальный закон распределения, симметричная копула, обмениваемость, коэффициент $\tau$-Кендалла, метод Монте--Карло, R-код.

Получение: 28 августа 2018 г. / Исправление: 23 октября 2018 г. Принятие: 12 ноября 2018 г. / Публикация онлайн: 18 ноября 2018 г.

Введение. Копула-функция является математическим объектом, инкапсулирующим в себе всю информацию о вероятностной структуре совместного распределения случайных переменных. Поэтому копулы применяются как для моделирования статистических зависимостей между случайными переменными, так и для построения новых совместных распределений. K настоящему времени выделено несколько конструктивных типов копул: эллиптические, архимедовы, креативные [1]. Эти копулы традиционно применяют для моделирования реальных статистических зависимостей случайных величин. Однако исследователи не оставляют попыток конструирования новых семейств копул. Так, в последнее десятилетие были предложены конические копулы [2], новые обобщения класса двумерных копул Родригеса-Лаллена и Убеда-Флорес (J. A. Rodríguez-Lallena, M. Úbeda-Flores) [3-5].

Новую копулу можно построить из многомерного распределения случайных величин, используя следствие из теоремы Шкляра (E. Sklar) [6, p. 22, Corollary 2.3.7], [7]. Примеры копул, извлеченных из новых совместных распределений, можно найти в работах [8-12].

Любая копула позволяет описывать зависимость между маргиналами в среднем и асимптотическую зависимость в хвостах совместного распределения. Для характеристики зависимости между маргиналами применяют коэффициент ранговой корреляции $\tau$-Кендалла (M. Kendall). Целью данной статьи является исследование свойств новой копулы, построенной из двупараметрического распределения односторонних статистик Граббса (F. Grubbs), возникающего в случае, когда присутствующее в выборке аномальное наблюдение отличается от остальных наблюдений величиной дисперсии.

Содержание статьи организовано следующим образом. В разделе 1 содержится обзор свойств совместного и маргинальных двупараметрических распределений односторонних статистик Граббса. В разделе 2 из совместного двупараметрического распределения односторонних статистик Граббса извлекается двупараметрическая копула Граббса, описывается алгоритм моделирования значений из копулы, а также доказывается ее симметричность. В разделе 3 описывается алгоритм оценивания коэффициента ранговой корреляции $\tau$-Кендалла копулы Граббса и исследуется влияние параметров копулы на его величину. В заключении рассматриваются возможные приложения полученных результатов.

1. О двупараметрическом совместном распределении статистик Граббса. Рассмотрим случайный набор из $n$ наблюдений: $X_{1}, X_{2}, \ldots, X_{n-1}$, $X_{n}$ и построенный по нему вариационный ряд:

$$
X_{(1)} \leqslant X_{(2)} \leqslant \cdots \leqslant X_{(n-1)} \leqslant X_{(n)},
$$


где $X_{(j)}-j$-тая порядковая статистика $(j=\overline{1, n})$.

Пусть гипотеза $H_{0}$ состоит в том, что случайные наблюдения $X_{1}, X_{2}, \ldots$, $X_{n-1}, X_{n}$ извлечены из генеральной совокупности, имеющей нормальное $N\left(a, \sigma^{2}\right)$ распределение, где параметры $a$ и $\sigma^{2}$ предполагаются неизвестными. В качестве альтернативной гипотезы $H_{1}$ рассмотрим случай, когда какиелибо $(n-1)$ наблюдения извлечены из генеральной совокупности с нормальным $N\left(a, \sigma^{2}\right)$ распределением, а одно из $n$ наблюдений (неизвестно какое по номеру) пришло из генеральной совокупности с нормальным $N\left(a+\alpha \sigma, \nu \sigma^{2}\right)$ распределением. Таким образом, аномальное наблюдение (выброс) $X_{\text {out }}$ отличается от остальных наблюдений параметром сдвига $\alpha$ и параметром масштаба $\nu>0$, а номер выброса в выборке неизвестен.

В качестве частного случая альтернативы $H_{1}$ рассмотрим гипотезу $H_{2}$, описывающую ситуацию, когда выброс $X_{\text {out }}$ отличается от остальных наблюдений только параметром масштаба $\nu>0$. Нулевую и альтернативные гипотезы можно формализовать следующим образом:

$H_{0}:(\alpha=0, \nu=1)$

$H_{1}:(\alpha \neq 0)$ или $(\alpha=0, \nu \neq 1)$;

$H_{2}:(\alpha=0, \nu \neq 1)$.

Возьмем односторонние статистики Граббса, т. е. экстремальные стьюдентизированные отклонения наблюдений от выборочного среднего, найденные по выборке из $n$ наблюдений:

$$
T_{n,(1)}=\frac{1}{S}\left(\bar{X}-X_{(1)}\right), \quad T_{n}^{(1)}=\frac{1}{S}\left(X_{(n)}-\bar{X}\right),
$$

где

$$
\bar{X}=\frac{1}{n} \sum_{i=1}^{n} X_{i}, \quad S^{2}=\frac{1}{n-1} \sum_{i=1}^{n}\left(X_{i}-\bar{X}\right)^{2} .
$$

Статистика $T_{n}^{(1)}$ была предложена Пирсоном и Чандра Секаром (E. S. Pearson, C. Chandra Sekar) [13] для проверки гипотезы о том, является ли наблюдение $X_{(n)}$ аномальным. Граббс впервые построил таблицу критических точек распределения статистики $T_{n}^{(1)}$ [14].

При справедливости гипотезы $H_{0}$ статистика $T_{n,(1)}$ имеет такой же закон распределения, что и статистика $T_{n}^{(1)}$ [14]:

$$
P\left(T_{n}^{(1)}<t \mid H_{0}\right)=P\left(T_{n,(1)}<t \mid H_{0}\right), \quad \forall t \in \mathbb{R} .
$$

Обозначим $F_{n}^{(1)}(t)=P\left(T_{n}^{(1)}<t \mid H_{0}\right)$. Закон распределения статистики $T_{n}^{(1)}$ (или $\left.T_{n,(1)}\right)$ может быть найден с помощью специальной рекурсивной процедуры, описанной в монографии [15, pp. 115-116]. В [15, p. 167], [16] с помощью данной процедуры построены рекурсивные соотношения для функции распределения статистики $T_{n}^{(1)}$ при справедливости гипотезы $H_{0}$ :

$$
F_{n}^{(1)}(t)=\left\{\begin{array}{cll}
0, & t \leqslant \frac{1}{\sqrt{n}}, & n \geqslant 2 ; \\
n \int_{1 / \sqrt{n}}^{t} F_{n-1}^{(1)}\left(g_{n}(x)\right) f_{T_{n}}(x) d x, & \frac{1}{\sqrt{n}}<t \leqslant \frac{n-1}{\sqrt{n}}, & n \geqslant 3 ; \\
1, & t>\frac{n-1}{\sqrt{n}}, & n \geqslant 2 ;
\end{array}\right.
$$


где

$$
\begin{gathered}
g_{n}(x)=\frac{n}{n-1} x\left(\frac{n-1}{n-2}\left(1-\frac{n}{(n-1)^{2}} x^{2}\right)\right)^{-1 / 2}, \quad|x|<\frac{n-1}{\sqrt{n}} ; \\
f_{T_{n}}(x)=\left\{\begin{array}{cl}
\frac{1}{n-1} \sqrt{\frac{n}{\pi}} \Gamma\left(\frac{n-1}{2}\right) / \Gamma\left(\frac{n-2}{2}\right)\left(1-\frac{n}{(n-1)^{2}} x^{2}\right)^{(n-4) / 2}, & |x|<\frac{n-1}{\sqrt{n}} ; \\
0, & |x| \geqslant \frac{n-1}{\sqrt{n}},
\end{array}\right.
\end{gathered}
$$

$\Gamma(x)$ - гамма-функция Эйлера.

Обозначим через $\Lambda_{n}\left(t_{1}, t_{2}\right)=P\left(T_{n,(1)}<t_{1}, T_{n}^{(1)}<t_{2} \mid H_{0}\right)$ совместную функцию распределения статистик Граббса в случае справедливости гипотезы $H_{0}$. В [17] доказано, что в случае $n>2$ функция $\Lambda_{n}\left(t_{1}, t_{2}\right)$ может быть описана следующим рекурсивным соотношением:

$$
\Lambda_{n}\left(t_{1}, t_{2}\right)=\left\{\begin{array}{cr}
F_{n}^{(1)}\left(t_{2}\right), & t_{1} \geqslant \frac{n-1}{\sqrt{n}} ; \\
F_{n}^{(1)}\left(t_{1}\right), & t_{2} \geqslant \frac{n-1}{\sqrt{n}} ; \\
n \int_{1 / \sqrt{n} \Lambda_{n-1}^{t_{2}}\left(\rho_{n}\left(t_{1},-x\right), g_{n}(x)\right) f_{T_{n}}(x) d x,}\left(t_{1}, t_{2}\right) \in \Delta_{n} ; \\
0, \quad\left(t_{1}, t_{2}\right) \notin \Delta_{n}, t_{1}<\frac{n-1}{\sqrt{n}}, t_{2}<\frac{n-1}{\sqrt{n}},
\end{array}\right.
$$

где $F_{n}^{(1)}(t)$ вычисляется по формуле $(1)$;

$$
\rho_{n}(t, z)=\left(t+\frac{z}{n-1}\right)\left(\frac{n-1}{n-2}\left(1-\frac{n}{(n-1)^{2}} z^{2}\right)\right)^{-1 / 2}, \quad|z|<\frac{n-1}{\sqrt{n}}
$$

функции $g_{n}(x)$ и $f_{T_{n}}(x)$ вычисляются по формулам $(2)$ и (3) соответственно;

$$
\Delta_{n}=\left[1 / \sqrt{n}<t_{1}<(n-1) / \sqrt{n} ; 1 / \sqrt{n}<t_{2}<(n-1) / \sqrt{n}\right] .
$$

В случае $n=2$ имеет место равенство [17]

$$
\Lambda_{2}\left(t_{1}, t_{2}\right)= \begin{cases}1, & \left(t_{1}, t_{2}\right) \in \Delta_{2} \\ 0, & \left(t_{1}, t_{2}\right) \notin \Delta_{2}\end{cases}
$$

где $\Delta_{2}=\left[1 / \sqrt{2}<t_{1}<\infty ; 1 / \sqrt{2}<t_{2}<\infty\right]$.

Предположим теперь, что верна альтернативная гипотеза $H_{1}$. Обозначим

$$
\widetilde{T}_{n}=\frac{1}{S}\left(X_{\text {out }}-\bar{X}\right)
$$

Случайная величина $\widetilde{T}_{n}$ является стьюдентизированным отклонением выброса $X_{\text {out }}$ от среднего, найденным по выборке объема $n$.

Обозначим через $f_{\widetilde{T}_{n}}(\cdot)$ - плотность распределения вероятностей случайной величины $\widetilde{T}_{n}$. Закон распределения случайной величины $\widetilde{T}_{n}$ был найден в [18] для случая, когда верна гипотеза $H_{1}$. В [19] доказано, что для вычисления плотности $f_{\widetilde{T}_{n}}(\cdot)$ может быть использована функция Әрмита, так что

$$
f_{\widetilde{T}_{n}}(t ; \alpha, \nu)=\beta_{n}(t ; \alpha, \nu) \cdot f_{T_{n}}(t) .
$$


Здесь $f_{T_{n}}(\cdot)$ вычисляется согласно $(3)$, а функция $\beta_{n}(\cdot)$ выражается через функцию Эрмита следующим образом:

$$
\beta_{n}(t ; \alpha, \nu)=\frac{c_{n}}{q_{n}^{(n-1) / 2}(t)} H_{-n+1}\left(-\frac{t \mu}{\sqrt{2 q_{n}(t)}}\right),
$$

где

$$
\begin{gathered}
c_{n}=\frac{2^{n-1}(n-1)^{n-1}}{\sqrt{\pi} n^{(n-1) / 2}} \eta^{(n-2) / 2} e^{-\mu^{2} / 2} \Gamma\left(\frac{n}{2}\right), \\
\mu=\alpha \sqrt{\frac{n-1}{1+\nu(n-1)}}, \quad \eta=\frac{1+\nu(n-1)}{n}, \quad q_{n}(t)=\eta \frac{(n-1)^{2}}{n}+(1-\eta) t^{2} ;
\end{gathered}
$$

функция Әрмита с отрицательным целым $k$ имеет вид

$$
H_{k}(z)=\frac{1}{\Gamma(-k)} \int_{0}^{\infty} e^{-\xi^{2}-2 z \xi} \xi^{-k-1} d \xi .
$$

Обозначим

$$
\begin{gathered}
G_{n,(1)}(t ; \alpha, \nu)=P\left(T_{n,(1)}<t \mid H_{1}\right), \\
G_{n}^{(1)}(t ; \alpha, \nu)=P\left(T_{n}^{(1)}<t \mid H_{1}\right), \\
\Upsilon_{n}\left(t_{1}, t_{2} ; \alpha, \nu\right)=P\left(T_{n,(1)}<t_{1}, T_{n}^{(1)}<t_{2} \mid H_{1}\right) .
\end{gathered}
$$

В [20] доказано, что для $n>2, \forall \alpha \in \mathbb{R}$ и $\nu>0$ справедливы следующие утверждения:

$$
G_{n}^{(1)}(t ; \alpha, \nu)=G_{n,(1)}(t ;-\alpha, \nu),
$$

$$
\begin{aligned}
& \quad 0, \quad 0 \leqslant t \leqslant \frac{1}{\sqrt{n}} ; \\
& G_{n,(1)}(t ; \alpha, \nu)=\left\{\begin{array}{cr}
\int_{-t}^{(n-1) / \sqrt{n}} F_{n-1}^{(1)}\left(\rho_{n}(t,-x)\right) f_{\widetilde{T}_{n}}(x ; \alpha, \nu) d x, & \frac{1}{\sqrt{n}}<t<\frac{n-1}{\sqrt{n}} ; \\
1, & t \geqslant \frac{n-1}{\sqrt{n}},
\end{array}\right. \\
& \Upsilon_{n}\left(t_{1}, t_{2} ; \alpha, \nu\right)=\left\{\begin{array}{cr}
G_{n}^{(1)}\left(t_{2} ; \alpha, \nu\right), & t_{1} \geqslant \frac{n-1}{\sqrt{n}} ; \\
G_{n,(1)}\left(t_{1} ; \alpha, \nu\right), & t_{2} \geqslant \frac{n-1}{\sqrt{n}} ; \\
\int_{-t_{1}}^{t_{2}} \Lambda_{n-1}\left(\rho_{n}\left(t_{1},-x\right), \rho_{n}\left(t_{2}, x\right)\right) f_{\widetilde{T}_{n}}(x ; \alpha, \nu) d x, \quad\left(t_{1}, t_{2}\right) \in \Delta_{n} ; \\
0, & \left(t_{1}, t_{2}\right) \notin \Delta_{n}, t_{1}<\frac{n-1}{\sqrt{n}}, t_{2}<\frac{n-1}{\sqrt{n}},
\end{array}\right.
\end{aligned}
$$

где функции распределения $F_{n-1}^{(1)}(\cdot), \Lambda_{n-1}^{(1)}(\cdot)$ и $f_{\widetilde{T}_{n}}(\cdot)$ определены согласно формулам $(1),(4)$ и $(7)$ соответственно, функция $\rho_{n}(\cdot)$ - согласно формуле (5), область $\Delta_{n}$ определена согласно (6).

В случае $n=2$ для $\forall \alpha \in \mathbb{R}$ и $\nu>0$ справедливы равенства

$$
G_{2}^{(1)}(t ; \alpha, \nu)=G_{2,(1)}(t ; \alpha, \nu)=F_{2}^{(1)}(t), \quad \Upsilon_{2}\left(t_{1}, t_{2} ; \alpha, \nu\right)=\Lambda_{2}\left(t_{1}, t_{2}\right) .
$$

Предположим теперь, что набор из $n$ наблюдений содержит выброс, который отличается от остальных наблюдений только величиной дисперсии. В 
таком случае параметр сдвига $\alpha=0$ и параметр масштаба $\nu>0$. Справедлива следующая

Теорема 1. Пусть гипотеза $H_{2}$ верна. Тогда для $\forall \nu>0$ плотность распределения вероятностей случайной величинь $\widetilde{T}_{n}$ имеет вид

$$
f_{\widetilde{T}_{n}}(t ; 0, \nu)=\frac{\sqrt{n}}{\sqrt{1+\nu(n-1)}\left(1+\frac{n(1-\nu) t^{2}}{(n-1)(1+\nu(n-1))}\right)^{(n-1) / 2}} \cdot f_{T_{n}}(t)
$$

где функиия $f_{T_{n}}(t)$ определена согласно (3).

Доказательство. Применим формулы (7) и (8) для случая $\alpha=0$. Тогда плотность $f_{\widetilde{T}_{n}}(\cdot)$ примет вид

$$
f_{\widetilde{T}_{n}}(t ; 0, \nu)=\frac{K_{n}}{q_{n}^{(n-1) / 2}(t)} \cdot f_{T_{n}}(t)
$$

где

$$
\begin{aligned}
& K_{n}=\frac{2^{n-1} \Gamma\left(\frac{n}{2}\right)}{\sqrt{\pi}} \cdot\left(\frac{n-1}{\sqrt{n}}\right)^{n-1} \eta^{(n-2) / 2} \cdot H_{-n+1}(0) \\
& q_{n}(t)=\eta \frac{(n-1)^{2}}{n}+(1-\eta) t^{2} ; \quad \eta=\frac{1+\nu(n-1)}{n}
\end{aligned}
$$

Используя (9), легко проверить, что $H_{-n+1}(0)=\frac{\Gamma\left(\frac{n-1}{2}\right)}{2 \Gamma(n-1)}$.

Теперь применим для гамма-функции Эйлера формулу двойного аргумента [21, с. 53]. В результате получим

$$
\Gamma(n-1)=\frac{1}{\sqrt{\pi}} 2^{n-2} \Gamma\left(\frac{n}{2}\right) \Gamma\left(\frac{n-1}{2}\right) .
$$

Поэтому $H_{-n+1}(0)=2^{1-n} \sqrt{\pi} / \Gamma\left(\frac{n}{2}\right)$. Тогда (14) примет вид

$$
K_{n}=\left(\frac{n-1}{\sqrt{n}}\right)^{n-1} \cdot \eta^{(n-2) / 2} .
$$

С учетом (15) и (16) выражение (13) примет вид (12), что и требовалось доказать.

Обозначим

$$
\begin{gathered}
S_{n,(1)}(t ; \nu)=P\left(T_{n,(1)}<t \mid H_{2}\right), \\
S_{n}^{(1)}(t ; \nu)=P\left(T_{n}^{(1)}<t \mid H_{2}\right), \\
\Theta_{n}\left(t_{1}, t_{2} ; \nu\right)=P\left(T_{n,(1)}<t_{1}, T_{n}^{(1)}<t_{2} \mid H_{2}\right) .
\end{gathered}
$$

Тогда справедливы следующие утверждения:

$$
S_{n,(1)}(t ; \nu)=G_{n,(1)}(t ; 0, \nu) ; S_{n}^{(1)}(t ; \nu)=G_{n}^{(1)}(t ; 0, \nu)
$$




$$
\begin{gathered}
\Theta_{n}\left(t_{1}, t_{2} ; \nu\right)=\Upsilon_{n}\left(t_{1}, t_{2} ; 0, \nu\right) \\
\Theta_{n}\left(t_{1}, t_{2} ; 1\right)=\Lambda_{n}\left(t_{1}, t_{2}\right) .
\end{gathered}
$$

Из (10) вытекает, что $G_{n}^{(1)}(t ; 0, \nu)=G_{n,(1)}(t ; 0, \nu)$. Поэтому $\forall t \in \mathbb{R}$ и $\nu>0$ имеем

$$
\begin{aligned}
S_{n,(1)}(t ; \nu) & =S_{n}^{(1)}(t ; \nu), \\
S_{n,(1)}(t ; 1) & =S_{n}^{(1)}(t ; 1)=F_{n}^{(1)}(t) .
\end{aligned}
$$

Следовательно, в условиях справедливости гипотезы $H_{2}$ статистика $T_{n,(1)}$ имеет такой же закон распределения, что и статистика $T_{n}^{(1)}$.

В [11] из совместного однопараметрического распределения статистик Граббса $T_{n,(1)}$ и $T_{n}^{(1)}$, найденного для случая справедливости гипотезы $H_{0}$, построена однопараметрическая копула-функция и исследованы ее свойства. В данной работе из совместного двупараметрического распределения $T_{n,(1)}$ и $T_{n}^{(1)}$, найденного для случая справедливости гипотезы $H_{2}$, будет построена двупараметрическая копула и исследованы ее свойства.

2. Построение двупараметрической копулы. Дадим определение копулы, следуя [6, p. 10, Definition 2.2.2].

ОпределЕниЕ. Двумерная копула-функция является отображением $C:[0,1]^{2} \rightarrow[0,1]$, которое обладает следующими свойствами:

$$
\begin{gathered}
C(0, v)=C(u, 0)=0, \quad \forall u, v \in[0,1] ; \\
C(u, 1)=u, \quad C(1, v)=v, \quad \forall u, v \in[0,1] ; \\
C\left(u_{1}, v_{1}\right)+C\left(u_{2}, v_{2}\right)-C\left(u_{1}, v_{2}\right)-C\left(u_{2}, v_{1}\right) \geqslant 0, \quad \text { если } u_{1} \leqslant u_{2}, v_{1} \leqslant v_{2} .
\end{gathered}
$$

Связь совместных распределений случайных переменных и копул описывает теорема Шкляра.

Пусть $Q(x, y)=P(X<x, Y<y)$ - совместная функция распределения случайных переменных $X$ и $Y$. Обозначим через $F(x)=P(X<x)$ и $G(y)=$ $=P(Y<y)$ - маргинальные функции распределений случайных переменных $X$ и $Y$.

Согласно теореме Шкляра [6, p. 18, Theorem 2.3.3], существует такая копула $C$, что

$$
Q(x, y)=C(F(x), G(y)) .
$$

Двупараметрическую копулу Граббса излечем из двумерного распределения $\Theta_{n}\left(t_{1}, t_{2} ; \nu\right)$, применив следствие из теоремы Шкляра [6, p. 22, Corollary 2.3.7], [7].

Обозначим через $s_{n}(\cdot)$ функцию, обратную функции частного распределения $S_{n,(1)}$. Тогда для $\forall \xi \in[0,1]$ и $t \in[1 / \sqrt{n},(n-1) / \sqrt{n}]$ справедливы следующие утверждения:

$$
S_{n,(1)}\left(s_{n}(\xi ; \nu) ; \nu\right)=\xi, \quad s_{n}\left(S_{n,(1)}(t ; \nu) ; \nu\right)=t .
$$

С учетом $(18) \forall \xi \in[0,1]$ и $t \in[1 / \sqrt{n},(n-1) / \sqrt{n}]$ имеем

$$
S_{n}^{(1)}\left(s_{n}(\xi ; \nu) ; \nu\right)=\xi, \quad s_{n}\left(S_{n}^{(1)}(t ; \nu) ; \nu\right)=t .
$$


Тогда $\forall(u, v)$ из единичного квадрата существует единственная копула-функция

$$
C_{G r}(u, v ; n, \nu):[0,1]^{2} \rightarrow[0,1]
$$

такая что

$$
C_{G r}(u, v ; n, \nu)=\Theta_{n}\left(s_{n}(u ; \nu), s_{n}(v ; \nu) ; \nu\right),
$$

где функция $\Theta_{n}\left(t_{1}, t_{2} ; \alpha, \nu\right)$ вычисляется согласно (11) и (17).

ЗАмечАниЕ. В определении (19) предполагается, что $n \geqslant 3$, т.к. в этом случае частные распределения $G_{n,(1)}$ и $G_{n}^{(1)}$ являются непрерывными и, следовательно, копула-функция Граббса $C_{G r}$ определена единственным образом [6, p. 18].

Теорема 2. Двупараметрическая копула Граббса является симметричной, m.е. $\forall(u, v) \in[0,1]^{2}$ имеет место равенство

$$
C_{G r}(u, v ; n, \nu)=C_{G r}(v, u ; n, \nu),
$$

где функиия $C_{G r}(\cdot)$ определена согласно (19).

Доказательств в. Действительно, с учетом (11) и $(17) \forall\left(t_{1}, t_{1}\right) \in \Delta_{n}$ можно записать

$$
\Theta_{n}\left(t_{1}, t_{2} ; \nu\right)=\int_{-t_{1}}^{t_{2}} \Lambda_{n-1}\left(\rho_{n}\left(t_{1},-x\right), \rho_{n}\left(t_{2}, x\right)\right) f_{\widetilde{T}_{n}}(x ; 0, \nu) d x
$$

где функция $f_{\widetilde{T}_{n}}(x ; 0, \nu)$ определена согласно $(12)$.

Замена переменной $z=-x$ приводит интеграл в (21) к виду

$$
\Theta_{n}\left(t_{1}, t_{2} ; \nu\right)=\int_{-t_{2}}^{t_{1}} \Lambda_{n-1}\left(\rho_{n}\left(t_{1}, z\right), \rho_{n}\left(t_{2},-z\right)\right) f_{\widetilde{T}_{n}}(-z ; 0, \nu) d z .
$$

В [17] доказано, что для $\forall(x, y) \in \Delta_{n}$ справедливо равенство

$$
\Lambda_{n}(x, y)=\Lambda_{n}(y, x)
$$

Кроме того, с учетом $(12) \forall|z|<\frac{n-1}{\sqrt{n}}$ можно записать

$$
f_{\widetilde{T}_{n}}(-z ; 0, \nu)=f_{\widetilde{T}_{n}}(z ; 0, \nu)
$$

Поэтому

$$
\Theta_{n}\left(t_{1}, t_{2} ; \nu\right)=\int_{-t_{2}}^{t_{1}} \Lambda_{n-1}\left(\rho_{n}\left(t_{2},-z\right), \rho_{n}\left(t_{1}, z\right)\right) f_{\widetilde{T}_{n}}(z ; 0, \nu) d z
$$

Откуда с учетом равенства (21) получим

$$
\Theta_{n}\left(t_{1}, t_{2} ; \nu\right)=\Theta_{n}\left(t_{2}, t_{1} ; \nu\right) .
$$

Теперь заметим, что с учетом $(19) \forall\left(t_{1}, t_{1}\right) \in \Delta_{n}$ можно записать

$$
\Theta_{n}\left(t_{1}, t_{2} ; \nu\right)=C_{G r}(u, v ; n, \nu)
$$


где $u=S_{n,(1)}\left(t_{1}\right)$ и $v=S_{n}^{(1)}\left(t_{2}\right)$.

Тогда равенство (22) примет вид (20), что и требовалось доказать.

Из симметричности копулы Граббса вытекает, что односторонние статистики Граббса $T_{n,(1)}$ и $T_{n}^{(1)}$ обладают свойством обмениваемости. Свойство обмениваемости для случайных величин $T_{n,(1)}$ и $T_{n}^{(1)}$ означает, что случайный вектор $\left(T_{n,(1)} ; T_{n}^{(1)}\right)$ имеет такое же распределение, что и случайный вектор $\left(T_{n}^{(1)} ; T_{n,(1)}\right)$ (см., например, [22, p. 2137]). Случайные величины, обладающие свойством обмениваемости, играют важную роль в таких областях статистики, как предельные законы, теория экстремальных значений, байесовские статистики и стохастические процессы [22, pp. 2136-2140].

На рис. 1-3 изображены скаттерплоты из копулы Граббса, построенные для ряда значений параметров $n$ и $\nu$. Скаттерплот представляет собой график смоделированных наблюдений из копулы Граббса. Точками скаттерплота являются наблюдаемые значения случайного вектора $U, V$, компоненты которого получены посредством равнометризации из разыгранных методом МонтеКарло значений статистик Граббса. Число разыгранных значений составило
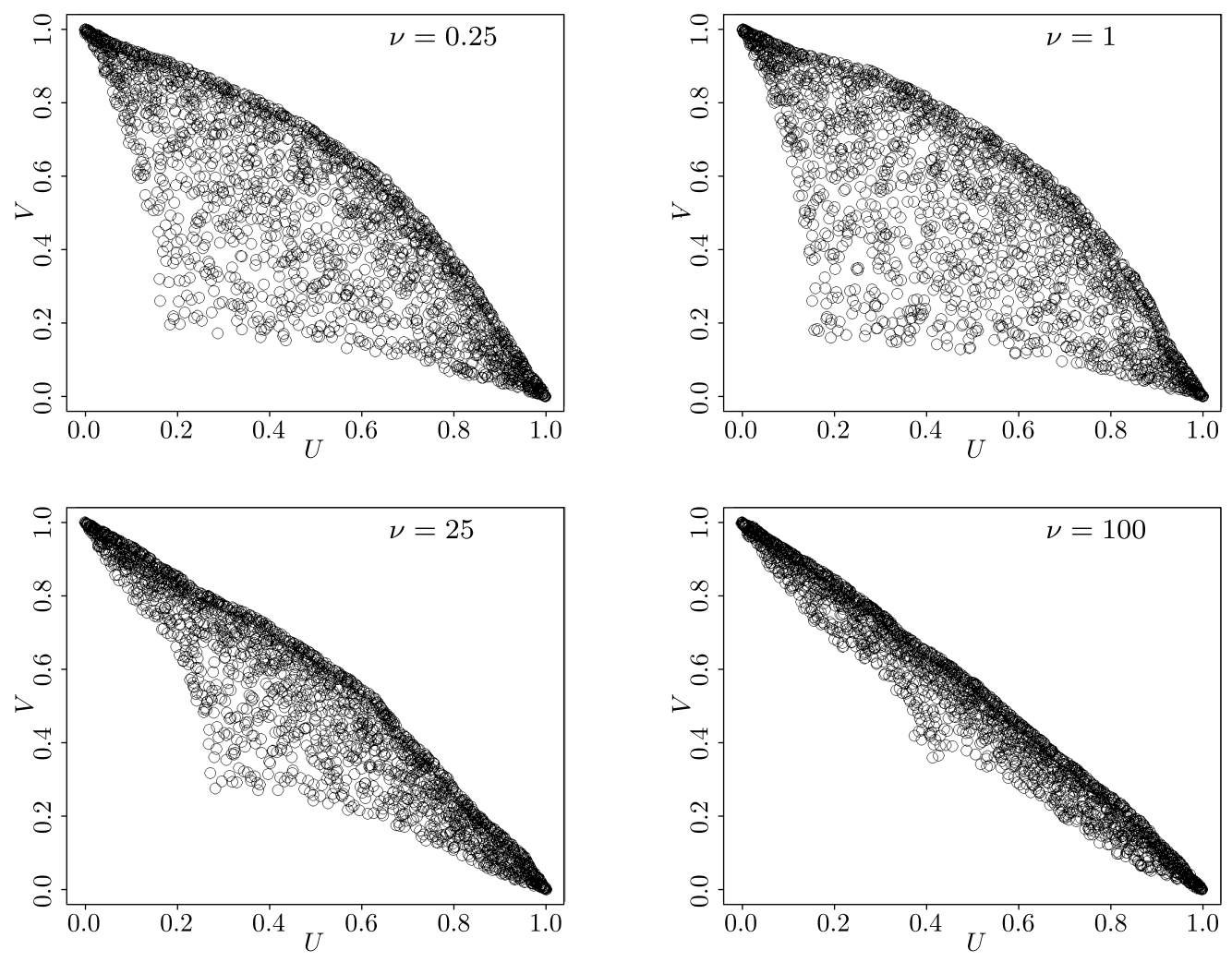

Рис. 1. Скаттерплоты из копулы Граббса в случае $n=4$ и различных значений параметра $\nu$ [Figure 1. Scatterplots from Grubbs' copula in the case $n=4$ and different values of the parameter $\nu$ ] 

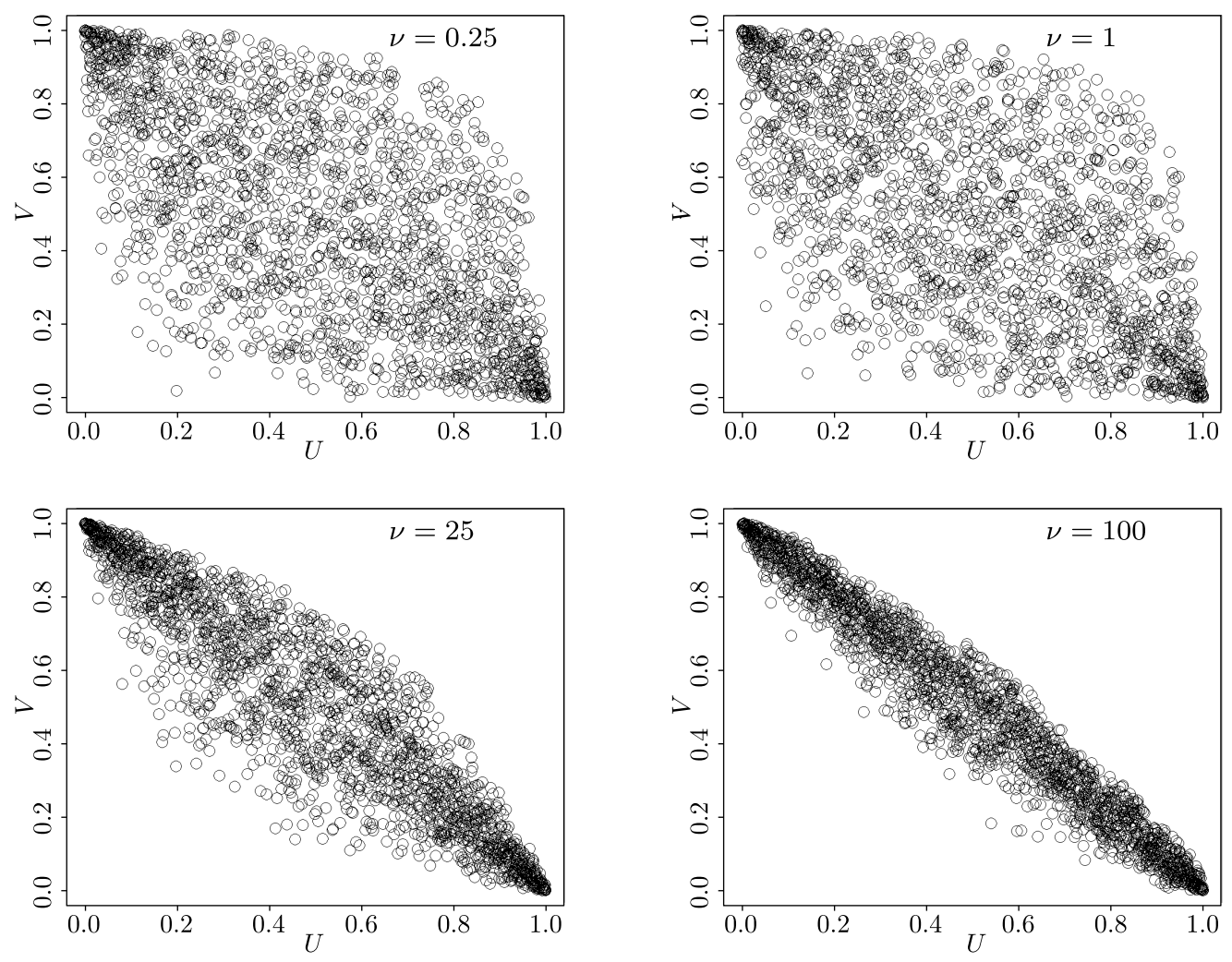

Рис. 2. Скаттерплоты из копулы Граббса в случае $n=7$ и различных значений параметра $\nu$

[Figure 2. Scatterplots from Grubbs' copula in the case $n=7$ and different values of the parameter $\nu$ ]

2000. Статистическое моделирование было реализовано в программной среде R [23]. R-код для построения скаттерплотов представлен в листинге 1.

Listing 1. Source code for building scatterplots

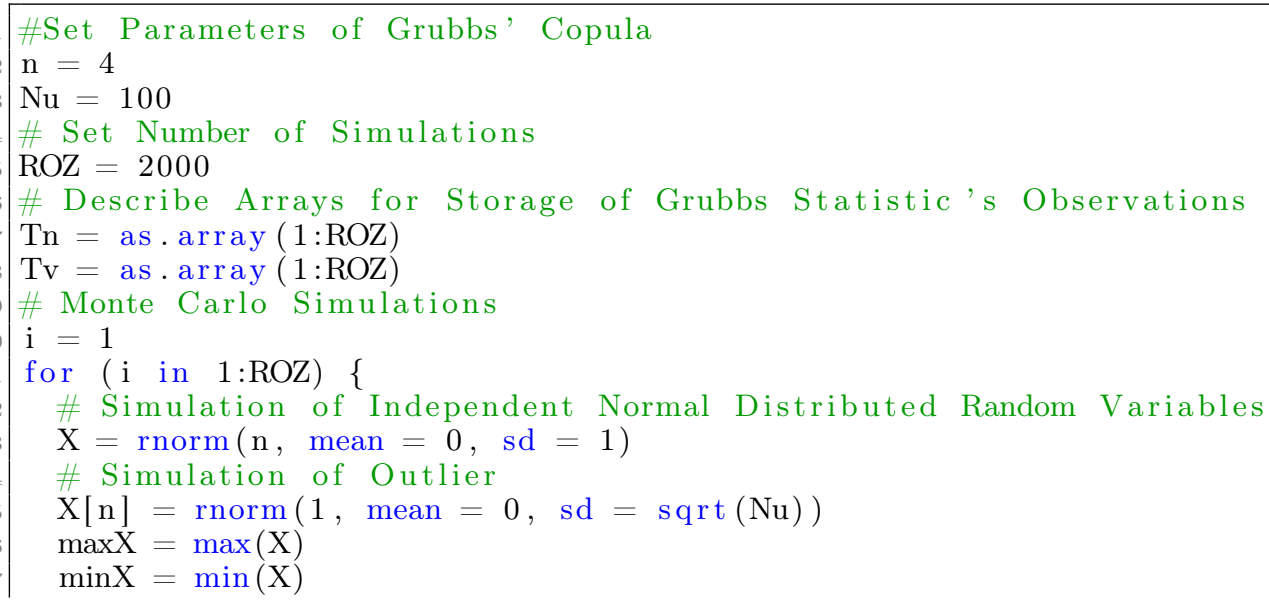


18 $\operatorname{meanX}=\operatorname{mean}(\mathrm{X})$

$\operatorname{sdX}=\operatorname{sd}(\mathrm{X})$

\# Calculation of Values of One-Sided Grubbs' Statistics

$\operatorname{Tn}[\mathrm{i}]=(\operatorname{mean} \mathrm{X}-\min \mathrm{X}) / \operatorname{sd} \mathrm{X}$

$\operatorname{Tv}[\mathrm{i}]=(\max \mathrm{X}-\operatorname{meanX}) / \operatorname{sdX}$

$\mathrm{i}=\mathrm{i}+1\}$

\# Archivation of Grubbs' Statistics Observations

$\mathrm{F} 1=\operatorname{ecdf}(\mathrm{Tn})$

$\mathrm{U}=\mathrm{ROZ} /(\mathrm{ROZ}+1) * \mathrm{~F} 1(\mathrm{Tn})$

$\mathrm{F} 2=\operatorname{ecdf}(\mathrm{Tv})$

$\mathrm{V}=\mathrm{ROZ} /(\mathrm{ROZ}+1) * \mathrm{~F} 2(\mathrm{Tv})$

\# Output of Scatterplot from Grubbs' Copula plot $(\mathrm{U}, \mathrm{V}$, type="p")
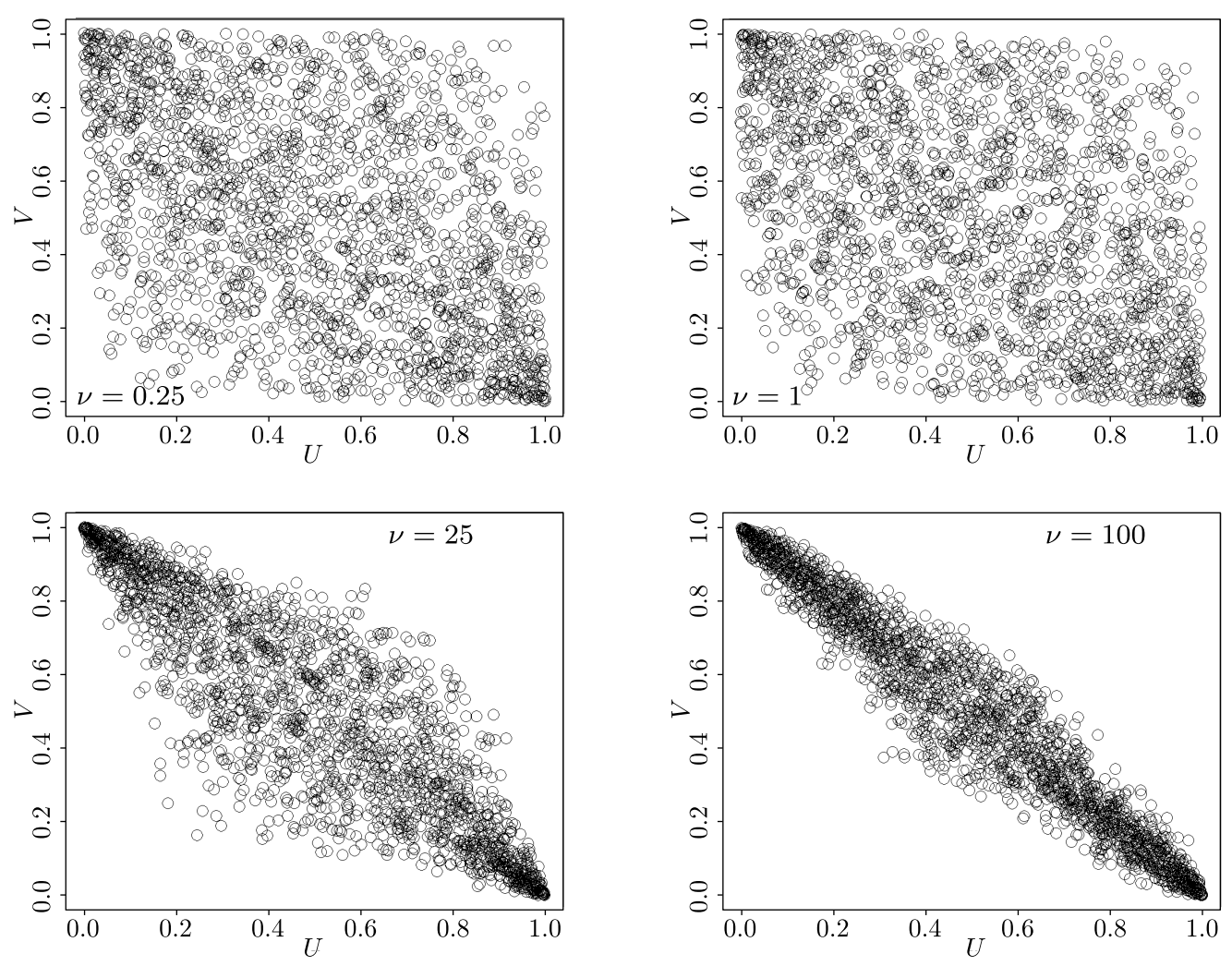

Рис. 3. Скаттерплоты из копулы Граббса в случае $n=15$ и различных значений параметра $\nu$

[Figure 3. Scatterplots from Grubbs' copula in the case $n=15$ and different values of the parameter $\nu$ ]

Из рис. 1-3 видно, что копула Граббса описывает отрицательную статистическую зависимость между случайными величинами. Можно также предположить, что параметры копулы $n$ и $\nu$ оказывают разнонаправленное влияние на силу зависимости между маргиналами, связанными копулой Граббса. Если параметр $\nu$ не меняется, то с ростом параметра $n$ наблюдается усиление разбросанности смоделированных точек. Такое поведение точек скаттерплота 
может указывать на уменьшение тесноты зависимости между маргиналами, связанными копулой Граббса. С другой стороны, рост параметра $\nu$ при неизменном $n$ сопровождается уменьшением разбросанности точек, что может отражать увеличение тесноты зависимости между маргиналами.

Для исследования влияния параметров копулы на силу взаимосвязи случайных переменных, заключенных в копулу Граббса, будем использовать коэффициент ранговой корреляции Кендалла ( $\tau$-Кендалла).

3. Исследование коэффициента $\tau$-Кендалла копулы Граббса. Дадим определение коэффициента ранговой корреляции $\tau$-Кендалла, следуя [6, p. 158]. Пусть $\left(X_{1}, Y_{1}\right)$ и $\left(X_{2}, Y_{2}\right)$ представляют собой независимые и одинаково распределенные случайные векторы, каждый с совместной функцией распределения $Q$. Тогда коэффициент $\tau$-Кендалла определяется как разность между вероятностью согласованности и вероятностью рассогласованности:

$$
\tau=\tau(X, Y)=P\left[\left(X_{1}-X_{2}\right)\left(Y_{1}-Y_{2}\right)>0\right]-P\left[\left(X_{1}-X_{2}\right)\left(Y_{1}-Y_{2}\right)<0\right] .
$$

Отметим следующие важные свойства коэффициента $\tau$-Кендалла, необходимые в дальнейшем [6, р. 158].

1. Коэффициент $\tau$-Кендалла симметричен, т.е. $\tau(X, Y)=\tau(Y, X)$.

2. $-1 \leqslant \tau(X, Y) \leqslant 1$.

3. Для двумерной случайной величины $(X, Y)$, совместное распределение которой описывается копулой $C$, коэффициент $\tau$-Кендалла равен

$$
\tau(X, Y)=4 \int_{0}^{1} \int_{0}^{1} C(u, v) \frac{\partial^{2} C(u, v)}{\partial u \partial v} d u d v-1 .
$$

Несмещенная непараметрическая оценка для коэффициента $\tau$-Кендалла, построенная по наблюдениям $\left(X_{i}, Y_{i}\right), i=1,2, \ldots, T$, имеет вид (см., напримep, [24])

$$
\hat{\tau}_{T}=\frac{2}{T(T-1)} \sum_{1 \leqslant i<j \leqslant T} \operatorname{sign}\left(X_{i}-X_{j}\right) \operatorname{sign}\left(Y_{i}-Y_{j}\right) .
$$

В работе [25] доказана асимптотическая нормальность оценки $\hat{\tau}_{T}$.

Применим (23) для двумерной случайной величины $\left(T_{n,(1)}, T_{n}^{(1)}\right)$, совместное распределение которой описывается двупараметрической копулой $C_{G r}$. Коэффициент $\tau$-Кендалла копулы Граббса будет зависеть от двух параметров: натурального $n$ и действительного $\nu>0$. С учетом формулы (23) можно записать

$$
\tau_{G r}(n, \nu)=4 \int_{0}^{1} \int_{0}^{1} C_{G r}(u, v ; n, \nu) \frac{\partial^{2} C_{G r}(u, v ; n, \nu)}{\partial u \partial v} d u d v-1,
$$

где $C_{G r}(\cdot)$ определяется согласно (19).

Сделаем замену $u=S_{n}^{(1)}(x ; \nu)$ и $v=S_{n}^{(1)}(y ; \nu)$ под знаком двойного интеграла в (25). В результате получим

$$
\tau_{G r}(n, \nu)=4 \int_{1 / \sqrt{n}}^{(n-1) / \sqrt{n}} \int_{1 / \sqrt{n}}^{(n-1) / \sqrt{n}} \Theta_{n}(x, y ; \nu) \frac{\partial^{2} \Theta_{n}(x, y ; \nu)}{\partial x \partial y} d x d y-1,
$$


где $\Theta_{n}(\cdot)$ определяется согласно (21).

Из (26) следует, что нахождение величины $\tau$-Кендалла для копулы $C_{G r}$ требует вычисления двойного интеграла от функции, заданной рекурсивно (см. формулы (21) и (4)). Это представляет собой сложную задачу. Поэтому в данной работе для исследования зависимости коэффициента $\tau$-Кендалла копулы Граббса от ее параметров был применен метод статистических испытаний (метод Монте-Карло).

Опишем процедуру оценивания коэффициента $\tau$-Кендалла копулы Граббса. Будем $T$ раз разыгрывать значения статистик $T_{n,(1)}$ и $T_{n}^{(1)}$ в условиях справедливости гипотезы $H_{2}$ и каждый раз вычислять по результатам розыгрыша оценку коэффициента $\tau$-Кендалла согласно (24). Будем повторять такие розыгрыши из $T$ значений статистик Граббса $L$ раз. В результате получим выборку из $L$ наблюдаемых значений оценки $\hat{\tau}_{T}: \hat{\tau}_{T ; 1}, \hat{\tau}_{T ; 2}, \ldots, \hat{\tau}_{T ; L}$. В качестве точечной оценки коэффициента $\tau$-Кендалла будем использовать оценку $\tau^{*}$, полученную как среднее арифметическое оценок $\hat{\tau}_{T ; 1}, \hat{\tau}_{T ; 2}, \ldots, \hat{\tau}_{T ; L}$, т. e.

$$
\tau^{*}=\frac{1}{L} \sum_{i=1}^{L} \hat{\tau}_{T ; i}
$$

Заметим, что оценка $\hat{\tau}_{T}$ является несмещенной оценкой коэффициента $\tau$ Кендалла. Легко проверить, что оценка $\tau^{*}$ также будет несмещенной.

Используя точечную оценку $\tau^{*}$, можно построить доверительный интервал для коэффициента $\tau$-Кендалла копулы Граббса. Для этого заметим, что оценка $\hat{\tau}_{T}$ является асимптотически нормальной. Поэтому при больших объемах выборки $T$ для построения интервальной оценки для $\tau_{G r}$ можно использовать статистику

$$
t=\frac{\left(\tau^{*}-\tau_{G r}(n, \nu)\right) \sqrt{L}}{S_{\hat{\tau}_{T}}}
$$

где

$$
S_{\hat{\tau}_{T}}=\sqrt{S_{\hat{\tau}_{T}}^{2}}, \quad S_{\hat{\tau}_{T}}^{2}=\frac{1}{L-1} \sum_{i=1}^{L}\left(\hat{\tau}_{T ; i}-\tau^{*}\right)^{2} ;
$$

оценка $\tau^{*}$ вычисляется по формуле $(27)$.

Согласно следствию из теоремы Фишера, статистика $t$ будет распределена по закону Стьюдента с $L-1$ степенями свободы [26, с. 221]. Тогда неравенство

$$
\tau^{*}-\Delta<\tau_{G r}(n, \nu)<\tau^{*}+\Delta
$$

будет выполняться с вероятностью $\gamma$. Здесь $\Delta=t_{(1+\gamma) / 2} S_{\hat{\tau}_{T}} / \sqrt{L} ; t_{(1+\gamma) / 2}-$ квантиль уровня $(1+\gamma) / 2 t$-распределения с $L-1$ степенями свободы.

Точность $\Delta$ интервальной оценки задает максимальное отклонение оценки $\tau^{*}$ от коэффициента $\tau_{G r}(n, \nu)$, которое можно гарантировать с вероятностью $\gamma$.

Для оценивания коэффициента $\tau$-Кендалла копулы Граббса использовался $R$-код, представленный в листинге 2 . 
Listing 2. Source code for estimating Kendall's tau coefficient

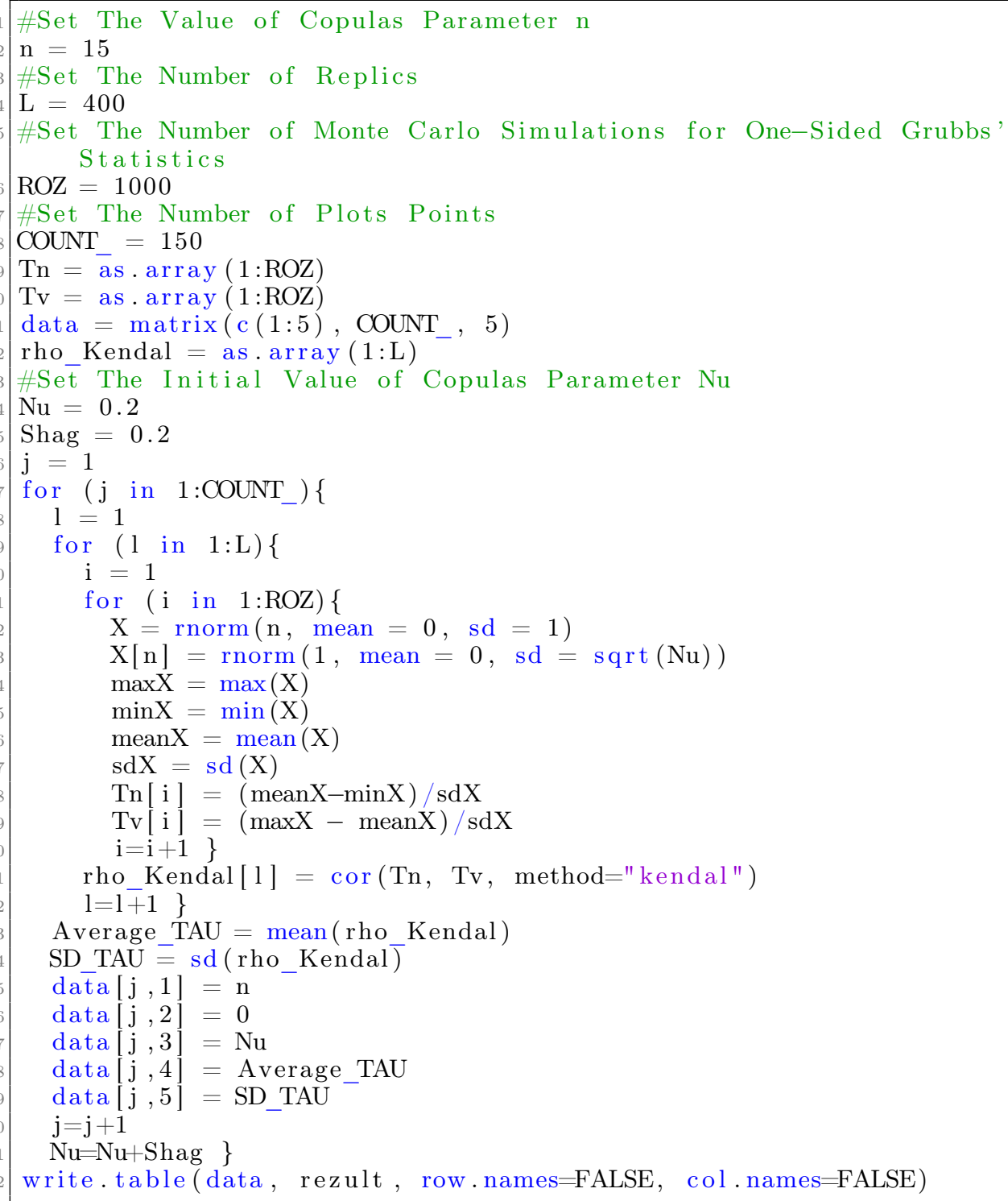

Статистическое моделирование оценки $\tau^{*}$ было проведено для значений параметров из диапазона $0.1 \leqslant \nu \leqslant 500$ и $4 \leqslant n \leqslant 50$. На рис. 4 и 5 приведены результаты оценивания коэффициента $\tau$-Кендалла копулы Граббса для ряда значений параметров $n$ и $\nu$ из указанного диапазона. Графики зависимости оценки $\tau^{*}$ построены для числа розыгрышей $T=1000$ и числа повторений $L=400$.

На рис. 6 и 7 приведены графики зависимости точности $\Delta$ интервальной оценки коэффициента $\tau_{G r}(n, \nu)$ от параметров $n$ и $\nu$ для случая, когда доверительная вероятность $\gamma=0.95$. Видно, что для исследуемого диапазона значений параметров $n$ и $\nu$ заведомо выполняется неравенство

$$
\left|\tau^{*}-\tau_{G r}(n, \nu)\right| \leqslant 0.0025
$$




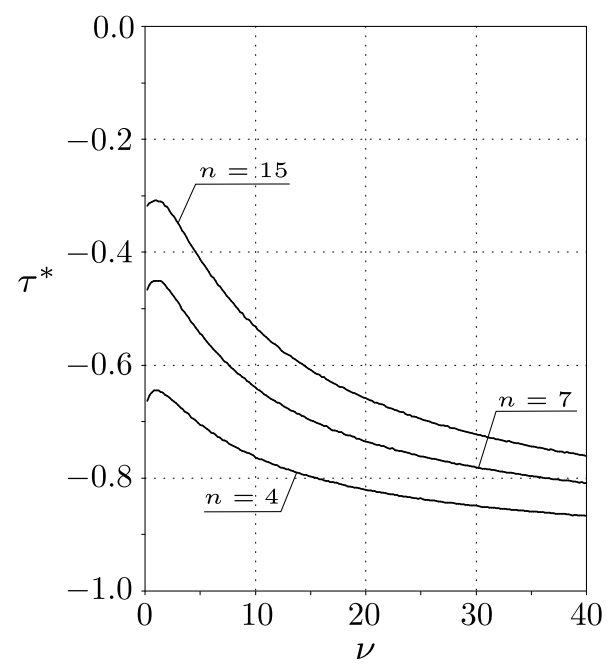

Рис. 4. Графики зависимости $\tau^{*}(\nu)$ для ряда значений параметра $n$

[Figure 4. Plots of Kendall's tau coefficient estimate depending on the parameter $\nu$ for different values of the parameter $n$ ]

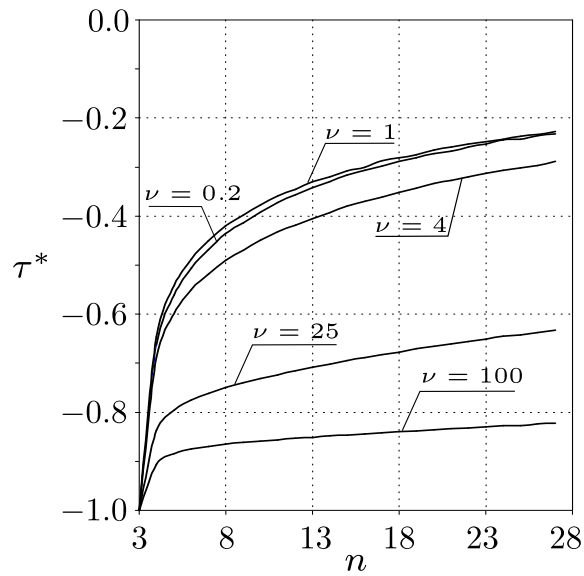

Рис. 5. Графики зависимости $\tau^{*}(n)$ для ряда значений параметра $\nu$

[Figure 5. Plots of Kendall's tau coefficient estimate depending on the parameter $n$ for different values of the parameter $\nu$ ]

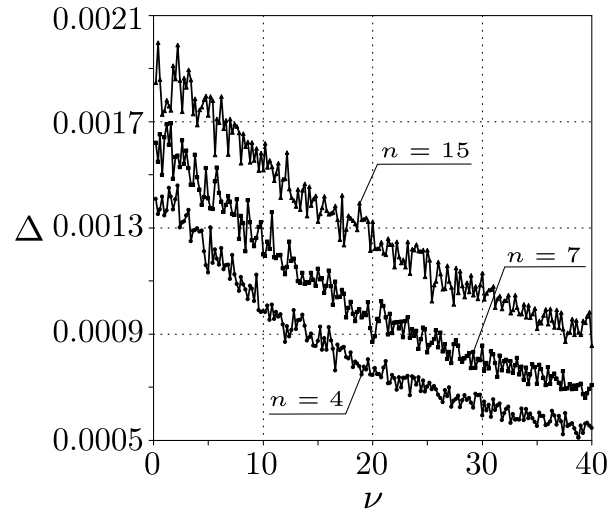

Рис. 6. Графики зависимости точности интервальной оценки $\Delta(\nu)$ для ряда значений параметра $n$ (доверительная вероятность 0.95)

[Figure 6. Plots of confidentional interval accuracy $\Delta$ depending on the parameter $\nu$ for different values of the parameter $n$ (confidence probability is 0.95 )]

Следовательно, выбор числа розыгрышей $T=1000$ и числа повторений $L=400$ гарантировал, что (28) выполняется с вероятностью, не меньшей 0.95.

Результаты статистического моделирования показали, что величина коэффициента $\tau$-Кендалла для копулы Граббса является отрицательной, что отражает существование отрицательной взаимосвязи между статистиками $T_{n,(1)}$ и $T_{n}^{(1)}$. Для исследованного диапазона значений параметров $0.1 \leqslant \nu \leqslant 500$ 


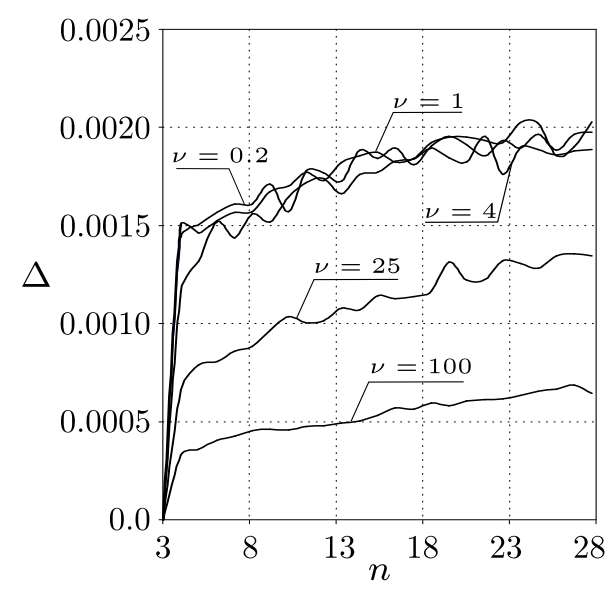

Рис. 7. График зависимости точности интервальной оценки $\Delta(n)$ для ряда значений параметра $\nu$ (доверительная вероятность 0.95)

[Figure 7. Plots of confidentional interval accuracy $\Delta$ depending on the parameter $n$ for different values of the parameter $\nu$ (confidence probability is 0.95 )]

и $4 \leqslant n \leqslant 50$ зависимость коэффициента $\tau_{G r}$ от параметра $\nu$ при фиксированной величине параметра $n$ не является монотонной. Рис. 4 демонстрирует это свойство коэффициента $\tau$-Кендалла копулы Граббса для случаев $n=4$, 10 и 15. При увеличении параметра $\nu$ до значений, близких к 1 , величина $\tau_{G r}$ уменьшается (по абсолютной величине), что отражает усиление силы взаимосвязи между маргиналами, заключенными в копулу Граббса. Дальнейший рост параметра $\nu$ приводит к росту коэффициента $\tau_{G r}$ (по абсолютной величине), что отражает уменьшение силы отрицательной взаимозависимости между маргиналами.

Из рис. 5 видно, как влияет параметр $n$ на коэффициент $\tau$-Кендалла при фиксированных величинах параметра $\nu$. Рост $n$ приводит к уменьшению (по абсолютной величине) коэффициента $\tau_{G r}$, что отражает уменьшение силы отрицательной взаимозависимости между маргиналами, заключенными в копулу Граббса.

Таким образом, параметры копулы $n$ и $\nu$ оказывают разнонаправленное влияние на величину коэффициента $\tau$-Кендалла копулы Граббса.

4. Заключение. Рассмотрен случай, когда в нормальной выборке объема $n$ одно наблюдение (неизвестно какое по номеру) является выбросом. Предполагалось, что дисперсия выброса в $\nu$ раз отличается от дисперсии остальных наблюдений. Исследовано двумерное распределение односторонних статистик Граббса, т. е. экстремальных стьдентизированных отклонений наблюдений от выборочного среднего, найденных по нормальной выборке с выбросом.

Из совместного распределения односторонних статистик Граббса построена двупараметрическая копула Граббса. Доказано, что двупараметрическая копула Граббса является симметричной, а односторонние статистики Граббса, совместное распределение которых описывается симметричной копулой Граббса, обладают свойством обмениваемости. Визуальный анализ скаттерплотов из копулы Граббса был проведен с помощью компьютерного моделирования, реализованного на языке R. Взаимное расположение точек скаттерплотов указывало на наличие отрицательных взаимосвязей между маргиналами, заключенными в копулу Граббса. Для измерения силы взаимосвязей между маргиналами копулы был использован коэффициент ранговой корреляции $\tau$-Кендалла. Оценивание коэффициента $\tau$-Кендалла было выполнено 
на языке R с помощью компьютерного моделирования. Найдено, что параметры копулы Граббса $n$ и $\nu$ оказывают разнонаправленное влияние на коэффициент ранговой корреляции. Так, рост параметра $n$ приводил к уменьшению (по абсолютной величине)коэффициента $\tau$-Кендалла, в то время как рост параметра $\nu$ до значений, значительно больших 1 , приводил к росту (по абсолютной величине) коэффициента $\tau$-Кендалла.

На наш взгляд, добавление к множеству традиционных копул новой симметричной копулы может обеспечить большие возможности для описания вероятностных структур реально существующих распределений.

Конкурирующие интересы. Мы заявляем, что у нас нет конфликта интересов в отношении авторства и публикации этой статьи.

Авторский вклад и ответственность. Мы несем полную ответственность за предоставление окончательной рукописи в печать. Каждый из нас одобрил окончательную версию рукописи.

Финансирование. Работа выполнялась без финансирования.

\section{Библиографический список}

1. Благовещенский Ю. Н. Основные элементы теории копул// Прикл. эконометрика, 2012. T. 26, № 2. C. 113-130.

2. Jwaid T., De Baets B., Kalicka J., Mesiar R. Conic aggregation functions // Fuzzy Sets and Systems, 2011. vol.167, no. 1. pp. 3-20. doi: j.fss.2010.07.004.

3. Rodríguez-Lallena J. A., Úbeda-Flores M. A new class of bivariate copulas // Statistics and Probability Letters, 2004. vol.66, no. 3. pp. 315-325. doi: 10.1016/j.spl.2003.09.010.

4. Kim J. M., Sungur E. A., Choi T., Heo T. Y. Generalized bivariate copulas and their properties // Model Assisted Statistics and Applications, 2011. vol.6, no. 2. pp. 127-136. doi: 10.3233/MAS-2011-0185.

5. Mesiar R., Najjari V. New families of symmetric/asymmetric copulas // Fuzzy Sets and Systems, 2014. vol.252, no. 1. pp. 99-110. doi: 10.1016/j.fss.2013.12.015.

6. Nelsen R. B. An Introduction to Copulas / Lecture Notes in Statistics. New York: SpringerVerlag, 2006. xiii +269 pp. doi : 10.1007/0-387-28678-0.

7. Фантаццини Д. Моделирование многомерных распределений с использованием копулафункций, I // Прикл. эконометрика, 2011. Т. 22, № 2. С. 98-134.

8. Ali Dolati, On Dependence Properties of Random Minima and Maxima// Communications in Statistics — Theory and Methods, 2008. vol.38, no.3. pp. 393-399. doi: 10.1080/ 03610920802213707.

9. Church C. The Asymmetric t-Copula with Individual Degrees of Freedom: A thesis submitted for the degree of MSc in Mathematical Finance. University of Oxford, Michaelmas, 2012. 48 pp., Available at http://www.maths.ox.ac.uk/system/files/legacy/12804/The_ Asymmetric_t-Copula_with_Individual_Degrees_of_Freedom.pdf (October 23, 2018).

10. Балаев А. И. Копула на основе многомерного $t$-распределения с вектором степеней свободы // Прикл. эконометрика, 2014. Т. 33, №1. С. 90-110.

11. Ширяева Л. К. О хвостовой зависимости для копула-функции Граббса // Изв. вузов. Матем., 2015. № 12. С. 66-83.

12. Kong-Sheng Zhang, Jin-Guan Lin, Pei-Rong Xu, A new class of copulas involved geometric distribution: Estimation and applications // Insurance: Mathematics and Economics, 2016. vol.66. pp. 1-10. doi: 10.1016/j.insmatheco.2015.09.008.

13. Pearson E. S., Chandra Secar C. The effciently of statistical tools and a criterion for the rejection of outlying observations // Biometrika, 1936. vol. 28, no. 3-4. pp. 308-320. doi : 10. 1093/biomet/28.3-4.308.

14. Grubbs F. Sample Criteria for Testing Outlying observations // Ann. Math. Statist., 1950. vol. 21, no. 1. pp. 27-58. doi: 10.1214/aoms/1177729885. 
15. Barnett V., Lewis T. Outliers in statistical data. Chichester: John Wiley \& Sons, 1984.

16. Zhang J., Keming Y. The null distribution of the likelihood-ratio test for one or two outliers in a normal sample// TEST, 2006. vol. 15, no. 1. pp. 141-150. doi: 10.1007/bf02595422.

17. Ширяева Л. К. О нулевом и альтернативном распределении статистики критерия наибольшего по абсолютной величине нормированного отклонения // Изв. вузов. Матем., 2014. № 10. С. $62-78$.

18. Ширяева Л. К. Вычисление мер мощности критерия Граббса проверки на один выброс // Сиб. журн. индустр. матем., 2010. Т. 13, № 4. С. 141-154.

19. Ширяева Л. К. Использование специальных функций Эрмита для исследования мощностных свойств критерия Граббса // Вестн. Сам. гос. техн. ун-та. Сер. Физ.-мат. науки, 2012. № 4(29). C. 131-145. doi : 10.14498/vsgtu1098.

20. Ширяева Л. К. О распределении статистик Граббса в случае нормальной выборки с выбросом // Изв. вузов. Матем., 2017. № 4. С. 84-101.

21. Янке Е., Эмде Ф., Лёш Ф. Специальные функиии. М.: Наука, 1977.

22. Galambos J. Exchangeability / Encyclopedia of statistical sciences. vol. 2; eds. S. Kotz, and N. L. Johnson. NY: Wiley, 1986.

23. R Core Team. R: A Language and Environment for Statistical Computing. R Foundation for Statistical Computing. Vienna, Austria, 2013, http://www.R-project.org/.

24. Ferguson S. T., Genest C., Hallin M. Kendall's tau for serial dependence // Canadian Journal of Statistics, 2000. vol. 28, no. 3. pp. 587-604. doi: 10.2307/3315967.

25. Grothe O., Schnieders J., Sigers J. Measuring association and dependence between random vectors // Journal of Multivariate Analysis, 2013. vol.123. pp. 96-110. doi:10.1016/j. jmva.2013.08.019.

26. Айвазян С. А., Мхитарян В. С. Прикладная статистика и основы эконометрики. Т. 1: Теория вероятностей и прикладная статистика. М.: Юнити-Дана, 2000. 


\title{
MSC: 62D05, 60E10
}

\section{On the some properties of a symmetric Grubbs' copula}

\author{
L. K. Shiryaeva, E. G. Repina
}

Samara State Economic University,

141, Sovetskoy Armii st., Samara, 443090, Russian Federation.

\begin{abstract}
We investigate one-sided Grubbs' statistics for a normal sample. Those statistics are standardized maximum and standardized minimum, i.e. studentized extreme deviation statistics. The two-parameter distribution of these statistics is considered, which arises when the one abnormal observation (outlier) differs from the other observations of its variance. We derive the formula for calculating the probability density function of studentized outlier deviation from sample average. A new two-parameter copula is extracted from the joint distribution of Grubbs' statistics. The Grubbs' copula is proved to be symmetric. As a result, one-sided Grubbs' statistics have the property of exchangeability. Computer simulation of scatterplots from Grubbs' copula is being performed. The scatterplot analysis shows that the Grubbs' copula describes the negative statistical dependence. To study the effect of the copula's parameters on the strength of this dependence, the estimation of the Kendall's tau rank correlation coefficient is performed. The estimation algorithm uses computer simulation and it is realized in the R-package. We find that the copula's parameters $n$ and $\nu>0$ have a multidirectional influence on the Kendall's tau coefficient. If we do not change the parameter $\nu$ then the growth of the parameter $n$ leads to a decrease (in absolute value) of the Kendall's tau coefficient, which reflects a decrease in the relationship's strength between the marginals in Grubbs' copula. If we do not change the parameter $n$, then growth of the parameter $\nu$ to 1 leads to a decrease in the Kendall's tau coefficient (in absolute value), which reflects a decrease in the strength of the relationship. Further growth of the parameter $\nu$ leads to an increase in the Kendall's tau coefficient (in absolute value), which reflects increased negative interdependence between the marginals.
\end{abstract}

Keywords: joint distribution function of maximum and standardized minimum, outlier, normal distribution, symmetric copula, exchangeability, Kendall's tau coefficient, Monte Carlo method, R-code.

\section{Research Article}

๖ () (7) The content is published under the terms of the Creative Commons Attribution 4.0 International License (http://creativecommons.org/licenses/by/4.0/)

Please cite this article in press as:

Shiryaeva L. K., Repina E. G. On the some properties of a symmetric Grubbs' copula, Vestn. Samar. Gos. Tekhn. Univ., Ser. Fiz.-Mat. Nauki [J. Samara State Tech. Univ., Ser. Phys. Math. Sci.], 2018, vol. 22, no. 4, pp. 714-734. doi:10.14498/vsgtu1640 (In Russian).

\section{Authors' Details:}

Ludmila K. Shiryaeva (10) http://orcid.org/0000-0003-2890-1786

Cand. Phys. \& Math. Sci.; Associate Professor; Dept. of Statistics and Econometrics; e-mail: Shiryeva_LK@mail.ru

Eugeniya G. Repina (D) http://orcid.org/0000-0001-7151-3266

Cand. Econom. Sci.; Associate Professor; Dept. of Statistics and Econometrics; e-mail: violet261181@mail.ru 
Received: 28 ${ }^{\text {th }}$ August, 2018 / Revised: 23 ${ }^{\text {rd }}$ October, 2018/

Accepted: $12^{\text {th }}$ November, $2018 /$ First online: $18^{\text {th }}$ November, 2018

Competing interests. We declare that we have no conflicts of interest in the authorship and publication of this article.

Authors' contributions and responsibilities. Each author has participated in the article concept development and in the manuscript writing. The authors are absolutely responsible for submitting the final manuscript in print. Each author has approved the final version of manuscript.

Funding. This research received no specific grant from any funding agency in the public, commercial, or not-for-profit sectors.

\section{References}

1. Blagoveshchenskii Yu. N. Basics of copula's theory, Applied Econometrics, 2012, vol. 26, no. 2, pp. 113-130 (In Russian).

2. Jwaid T., De Baets B., Kalicka J., Mesiar R. Conic aggregation functions, Fuzzy Sets and Systems, 2011, vol. 167, no.1, pp. 3-20. doi: j.fss.2010.07.004.

3. Rodríguez-Lallena J. A., Úbeda-Flores M. A new class of bivariate copulas, Statistics and Probability Letters, 2004, vol.66, no. 3, pp. 315-325. doi: 10.1016/j.spl.2003.09.010.

4. Kim J. M., Sungur E. A., Choi T., Heo T. Y. Generalized bivariate copulas and their properties, Model Assisted Statistics and Applications, 2011, vol.6, no. 2, pp. 127-136. doi: 10.3233/MAS-2011-0185.

5. Mesiar R., Najjari V. New families of symmetric/asymmetric copulas, Fuzzy Sets and Systems, 2014, vol.252, no. 1, pp. 99-110. doi: 10.1016/j.fss.2013.12.015.

6. Nelsen R. B. An Introduction to Copulas, Lecture Notes in Statistics. New York, SpringerVerlag, 2006, xiii+269 pp. doi : 10.1007/0-387-28678-0.

7. Fantazzini D. Analysis of Multidimensional Probability Distributions with Copula Functions, I, Applied Econometrics, 2011, vol.22, no. 2, pp. 98-134 (In Russian).

8. Ali Dolati, On Dependence Properties of Random Minima and Maxima, Communications in Statistics - Theory and Methods, 2008, vol.38, no. 3, pp. 393-399. doi: 10.1080/ 03610920802213707.

9. Church C. The Asymmetric t-Copula with Individual Degrees of Freedom, A thesis submitted for the degree of MSc in Mathematical Finance. University of Oxford, Michaelmas, 2012, 48 pp., Available at http://www.maths.ox.ac.uk/system/files/legacy/12804/The Asymmetric_t-Copula_with_Individual_Degrees_of_Freedom.pdf (October 23, 2018).

10. Balaev A. I. The copula based on multivariate $f$-distribution with vector of degrees of freedom, Applied Econometrics, 2014, vol. 33, no. 1, pp. 90-110 (In Russian).

11. Shiryaeva L. K. On tail dependence for Grubbs' copula-function, Russian Math. (Iz. VUZ), 2015, vol. 59, no. 12, pp. 56-72. doi: 10.3103/S1066369X15120063.

12. Kong-Sheng Zhang, Jin-Guan Lin, Pei-Rong Xu, A new class of copulas involved geometric distribution: Estimation and applications, Insurance: Mathematics and Economics, 2016, vol. 66, pp. 1-10. doi: 10.1016/j.insmatheco.2015.09.008.

13. Pearson E. S., Chandra Secar C. The effciently of statistical tools and a criterion for the rejection of outlying observations, Biometrika, 1936, vol. 28, no. 3-4, pp. 308-320. doi: 10. 1093/biomet/28.3-4.308.

14. Grubbs F. Sample Criteria for Testing Outlying observations, Ann. Math. Statist., 1950, vol. 21, no. 1, pp. 27-58. doi: 10.1214/aoms/1177729885.

15. Barnett V., Lewis T. Outliers in statistical data. Chichester, John Wiley \& Sons, 1984. 
16. Zhang J., Keming Y. The null distribution of the likelihood-ratio test for one or two outliers in a normal sample, TEST, 2006, vol.15, no. 1, pp. 141-150. doi: 10.1007/bf02595422.

17. Shiryaeva L. K. On null and alternative distribution of statistics of two-side discordancy test for an extreme outlier, Russian Math. (Iz. VUZ), 2014, vol. 58, no. 10, pp. 52-66. doi: 10. 3103/S1066369X14100089.

18. Shiryaeva L. K. Calculation of power measures of Grabbs' criterion for checking for one outlier, Sib. Zh. Ind. Mat., 2010, vol. 13, no. 4, pp. 141-154 (In Russian).

19. Shiryaeva L. K. Using of special Hermite functions for investigation of power properties of Grubbs' criterion, Vestn. Samar. Gos. Tekhn. Univ., Ser. Fiz.-Mat. Nauki [J. Samara State Tech. Univ., Ser. Phys. Math. Sci.], 2012, no.4(29), pp. 131-145 (In Russian). doi: 10. 14498/vsgtu1098.

20. Shiryaeva L. K. On distribution of Grubbs' statistics in case of normal sample with outlier, Russian Math. (Iz. VUZ), 2017, vol.61, no. 4, pp. 72-88. doi: 10.3103/S1066369X17040107.

21. Jahnke E., Emde F., Lösch F. Tafeln höherer Funktionen [Tables of higher functions]. Stuttgart, B. G. Teubner Verlagsgesellschaft, 1960, xii +318 pp. (In German)

22. Galambos J. Exchangeability, In: Encyclopedia of statistical sciences, vol. 2; eds. S. Kotz, and N. L. Johnson. NY, Wiley, 1986.

23. R Core Team. $R$ : A Language and Environment for Statistical Computing. R Foundation for Statistical Computing. Vienna, Austria, 2013, http://www.R-project.org/.

24. Ferguson S. T., Genest C., Hallin M. Kendall's tau for serial dependence, Canadian Journal of Statistics, 2000, vol. 28, no. 3, pp. 587-604. doi : 10.2307/3315967.

25. Grothe O., Schnieders J., Sigers J. Measuring association and dependence between random vectors, Journal of Multivariate Analysis, 2013, vol. 123, pp. 96-110. doi : 10.1016/j.jmva. 2013.08.019.

26. Aivazian S. A., Mkhitaryan V. S. Applied Statistics and Essentials of Econometrics, vol. 1, Probability Theory and Applied Statistics. Moscow, Yuniti-Dana, 2000 (In Russian). 